\title{
Zuviel Vertrauen? \\ Über Moral und Finanzen
}

\author{
BIRgER P. PRIDDAT
}

\section{Beyond Trust - On the Relationship of Morality and Finance}

Many authors do believe in the crisis of the banking system as a failure of trust relations. They are looking for new moral standards and better regulations. But they ignore the fact that most transactions of the toxic certificates were based on trust between suppliers and demanders. Trust doesn't seem to be a solution, but a part of the problem.

Keywords: Bankenkrise, Vertrauen, Risiko, Moral der Wirtscbaft

\section{Einführung}

Wir neigen dazu, die Finanzkrise als Vertrauenskrise zu beschreiben. Die Anleger haben ihr Vertrauen in die Seriosität der Banken verloren. Deren Geschäftskultur habe sich als unbalanciert und unentwickelt erwiesen, und sei nicht in der Lage gewesen, den Kunden die Beratung zukommen zu lassen, die Risiken sorgsam behandelte. Der deutsche Bundespräsident, selber ein ehemaliger Banker, ruft die Branche zu mehr Demut und Anstand auf.

Demut und Anstand sind eher moralische Begriffe. Oft werden auch Gier und Hybris als Ursachen der Finanzkrise ausgemacht. Damit wird unterstellt, dass es eigentlich ein $\mathrm{Maß}$ gäbe, das, eingehalten, die Krise nicht hätte entfalten lassen. Die moralische Kritik orientiert sich an Tugenden. Ist der Kapitalismus kulturell auf Tugendkataloge gegründet? Ist die Finanzkrise eine moralische oder Tugendkrise?

Es scheint so, dass der Anreiz, schnell hohe Profite zu machen, die Haltung zu einer angemessenen (und risikokompetenten) Geschäftskultur unterhöhlt hat. Das sind immer wieder vorkommende Gefährdungen in kapitalistischen Systemen, die gewöhnlich durch Wettbewerb balanciert werden. Wenn alle aber sich unilateral in eine Richtung bewegen, fallen die Hebel der Konkurrenz aus; es entsteht ein gemeinsam geteiltes mentales Modell einer Welt des Gewinnwachstums, das ,nicht auszunutzen' gleichsam als fahrlässig angesehen wird. Es ist ein Kommunikationsrauschen, in dem nur rosa Farben gesehen werden, begleitet von einem Glauben, dass der Strom schon nicht ausfallen werde. Dass die in den Geschäften versteckten Risiken aber die Energie fressen, bleibt im Rauschen unentdeckt: Ein Geheimnis, das niemand hören will -

Prof. Dr. Birger P. Priddat, Universität Witten/Herdecke, Alfred Herrhausenstr. 50, D-58448 Witten, Tel.: +49-(0)2302-926-530, E-Mail: birger.priddat@uni-wh.de, Forschungsschwerpunkte: Institutionenökonomie, Wirtschaftsethik, Theoriegeschichte der Ökonomie, Politische Ökonomie, Wirtschaft und Kommunikation. 
obwohl es genügend kluge Kommentare vieler Ökonomen gab, und obwohl eine gewisse Kenntnis der Geschichte der Ökonomie, insbesondere ihrer Finanzkrisen, eine andere Sensibilität hätte entwickeln lassen können. Macht sich hier der Mangel der Ausbildung moderner Ökonomen bemerkbar, die geschichtliche Erinnerung nicht kennen?

Vielfach waren die Anleger selber extrem risikofreudig, aber nicht, weil sie unmoralisch oder tugendlos handelten, sondern weil sie die Profitversprechen der Anlageberater vor dem Hintergrund akzeptierten, die Bank sei so groß, also sicher. Nur vor dem Hintergrund dieser sublunar mitlaufenden Gewährleistungsmetaphysik sind die Risiken zu verstehen, die die Anleger eingingen: Sie vertrauen der Kompetenz der Banker und der Gewissheit, große Häuser könnten gar nicht untergehen. Aber auch die Schwierigkeit, Risiken einschätzen zu können, spielt mit hinein:
„Deshalb nehmen wir für einen potentiell hohen Gewinn viel mehr Verlust in Kauf, als es auf der Grundlage der Wahrscheinlichkeit seines Eintretens gerecht- fertigt wäre. Und aus demselben Grund sind wir bereit, für einen Zinspunkt mehr die Bank zu wechseln, selbst wenn dies eine Vervielfachung der Verlust- wahrscheinlichkeit bedeutet.
Um überhaupt eine Risikoeinschätzung vornehmen zu können, werden meist Heuristiken (einfache Faustregeln) angewandt, wie zum Beispiel ein vermeintli- cher Trend bei der Wertentwicklung in der Vergangenheit oder die Einschät- zung von Experten. Diese menschliche Schwäche öffnet natürlich Tür und Tor für gezielte Einflussnahmen und Manipulationen. Dies gilt umso mehr, wenn das Risiko verschleiert wird - wie bei den Finanzderivaten, die wesentlich zur jetzigen Krise beigetragen haben. Und wenn die Experten (hier: die Rating- Agenturen) mit den Anbietern unter einer Decke stecken, ist der Kunde völlig verloren" (Strack 2008).

Investmentbanker wiederum wussten um die Risiken der strukturierten Papiere, die sie emittierten, hofften aber auf den Staat als, lender of last resort', weil es politisch unmöglich sei, das ganze Finanzsystem, käme es in die Krise, untergehen zu lassen. Die Banker wetteten auf den Teufel ihrer neoliberalen Grundeinstellung: auf den Staat. Karl Homann plädiert deshalb für mehr geregelten Wettbewerb. Bestes Beispiel für ungeregelten Wettbewerb sind demnach:

„die neuen Instrumente für den Finanzmarkt, die in den vergangenen Jahren auf den Markt kamen und naturgemäß noch keinem geeigneten Regelsystem unterlagen, zum Teil dienten sie wohl auch dazu, sich den bestehenden Bilanz- und Kontrollregeln zu entziehen.

Diese neuen Produkte dienten im Wettbewerb wie nicht anders zu erwarten allein dazu, Rendite und Wachstum zu steigern. Und ihr Erfolg sorgte für den entsprechenden Druck auf die Konkurrenz: Es blieb dem einzelnen Bankvorstand gar nichts anderes übrig als mitzumachen. Andernfalls wäre er entlassen worden, mit der Begründung, dass er die Chancen des Marktes nicht erkannt habe. Der scharfe Wettbewerb unter unzureichenden Regeln führte also in die kollektive Irrationalität, zum Zusammenbruch des Systems - und nicht ein irrationaler ,Herdentrieb'. Vermutlich hat manch ein Banker diese Folge vorhergesehen, konnte aber als Einzelner unter dem Druck des Wettbewerbs nichts dagegen ausrichten“ (Homann 2009). 
Tatsächlich wussten viele Banker um die Brisanz ihrer Geschäftspolitiken, hielten sich aber im Spiel mit der Meinung, weil sie kompetent wären, wüssten sie, wann man aussteigen müsste. Diese rationale Erwartung bezüglich ihrer eigenen Handlungsfähigkeit wurde im Wettbewerb um die größten Gewinnchancen nicht eingelöst. Niemand traute sich, als erster auszusteigen. Die „kollektive Irrationalität“, wie Homann es nennt, ist nicht mehr ökonomisch zu begründen, durch Abwägen von Nutzen und Risiken, sondern soziologisch: als Status- und Reputationswettbewerb. Wer ist alpha, wer beta?

Es entwickelt sich eine Atmosphäre im Markt, die nicht nur als Gewinneuphorie zu beschreiben ist, sondern in einen Statuswettbewerb übergeht: Wer ist der beste? Wer holt mehr heraus? Die Kollegen in der Bank - wie die Bankmanager gegeneinander beobachten sich auf diese reputative Auszeichnung. Bei Lehmann u. a. gab es täglich Listen über Gewinner und Verlierer. Forciert wird diese Reputations- oder Statuswettbewerbskultur durch

\begin{abstract}
„Anreizsysteme, die es Verkäufern und Käufern ersparen, das Risiko realistisch einzuschätzen. Dies ist vor allem dann der Fall, wenn die Entscheidung dem Handelnden selbst keinen Schaden zufügt. Kurzfristige Belohnungssysteme, die lediglich von der Höhe des Gewinns bestimmt werden, ohne bei Misserfolg einen Verlust spürbar zu machen, verhindern eine ausreichende Berücksichtigung des Risikos. Deshalb sind Prämien, die sich auf einen längeren Zeitraum erstrecken, viel besser geeignet, auch Verluste und somit das Risiko in die Entscheidung einfließen zu lassen" (Strack 2008).
\end{abstract}

Dass Risiken bestanden, kann keiner leugnen. Sie wurden ausgeblendet. Wir haben es mit zwei großen Sicherungsglaubenssätzen zu tun: (1) Große Banken können nicht untergehen und (2) notfalls rettet der Staat - (3) könnte man anfügen, Ratingagenturen sind irgendwie objektiv (vgl. u. a. Coval et al. 2009). Es sind Glaubenssätze, die man von Ökonomen meinte, niemals hören zu sollen (ich gehe davon aus, dass Banker Ökonomen sind). Alle drei Propositionen dämpfen das Risiko; es ist erstaunlich, wie viel ökonomische Kompetenz zusammen, im Schwarm, den volkswirtschaftlichen oder Systemeffekt zu ignorieren in der Lage ist. Vor allem aber wurde diesem System vertraut. Es ist zugleich erstaunlich, wie wenig dem eigenen Urteil noch vertraut wurde bzw. wie das eigene Urteil, das die Ökonomen als rational und unabhängig gelernt haben, in einem Modus kollektiven Irrens abgeschafft wurde. Ein Teil der Regelwerke, die jetzt erstellt werden, wird darin bestehen, „wie Staaten glaubwürdig machen können, dass Finanzinstitute im Fall des Falles nicht vom Staat herausgehauen werden" (Siebert 2008: Sp. 5). Wie stellt die Politik ihre Drohung glaubwürdig?

Nebenbei zeigt sich eine Unklarheit: Was betriebswirtschaftlich bedeutsam sein mag, ist es volkswirtschaftlich noch lange nicht. Das Gespür für den Unterschied zwischen privaten und öffentlichen Interessen ist verloren gegangen (vgl. Hellwig 2008). Allein von daher wird der Staat eine neue Rolle spielen. Der Markt hat keine Unbedenklichkeitsbescheinigung mehr. 


\section{Anlegervertrauen}

Kaum ein Käufer wird sich einem Angebot hoher Renditen verschließen, wenn es von einer Bank kommt, deren Beratung man schon viele Jahre erfolgreich in Anspruch genommen hat, deren Berater man persönlich kennt, zu der man Vertrauen aufgebaut hat, das durch Ratingagenturen bestätigt wird und von der öffentlichen Meinung, den Medien, oft auch von der Politik. Wenn das Vertrauen so über die Zeit gefestigt wurde, schlägt es nicht plötzlich in Risikoaversion um - weil das Vertrauen besteht. Es ist ein soziales Kapital der Bank/Kunden-Beziehungen geworden, das sich in solchen Erwartungssituationen besonders auszuzahlen scheint. Man ist - über das Vertrauen moralisch auf Erfolg gestimmt.

Das ist die besondere Paradoxie der Finanzkrise: Weil man vertraute, unterschätzte man das Risiko. Weil man das Risiko unterschätzte, wirkte sich das Vertrauen, die eigentlich hochwertige moral relation, als Krisenverstärker aus. Vertrauen, eine der Beschwörungsvokabeln guter Wirtschaft, anscheinend ein Gewährleistungsgarant, als Verstärker der Finanzkrise?

So sehr ein Anleger als ,gierig' erscheinen mag: Seine Gier ist keine Charakterschwäche, sondern angebotsgetrieben, durch die Kommunikation vertrauenswürdiger Akteure der Banken, auf deren Urteil man sich bisher immer verlassen konnte. Der Entschluss der Anleger, auf hohe Renditen einzugehen, entsteht im Vertrauensraum - ein sozialer Raum wechselseitiger Anerkennung, in dem sehr auf das Gute gehofft wird, weil man alles andere zu erwarten - vertrauensvoll - ausblendet. Erst auf dieser moralischen Basis scheinen die hohen Renditeangebote als legitimiert. Man geht nicht natürlich ins Risiko; es braucht eine Atmosphäre des Vertrauens und des Glaubens, dass dieses Vertrauen auch angesichts neuer Wachstumspotentiale gerechtfertigt ist.

Betrachten wir das Vertrauen genauer, ist es ratsam, zwischen einer alten und einer modernen Form zu unterscheiden. Die ,alte Form` ist die einer loyalen Bindung. Man geht ein Verpflichtungsverhältnis ein, auf das wechselseitig stark vertraut werden kann, mit hoher normativer Kraft. Verletzt einer die Vertrauens-Loyalität, hat das soziale Folgen: Ausschluss aus dem Netzwerk, der Familie, dem Clan. Die Mafia ist noch ein Beispiel für solche alten Formen. Wer das Vertrauen bricht, verletzt die Ehre. Es gilt ein sittlicher Kodex.

Modernes Vertrauen ist opportunistischer. Man vertraut, weiß aber zugleich, dass das Vertrauen enttäuscht oder ausgebeutet werden kann. Es ist eine lose Kopplung, keine starke normative Bindung. Man vertraut häufig auch darauf, dass andere Probleme klären, die man selber nicht entscheiden kann oder es als zu aufwendig empfindet. In diesem Sinne senkt Vertrauen die Komplexität oder zumindest die Transaktionskosten einer Vertragsbeziehung. Man delegiert Entscheidungen, indem man vertraut: meistens weniger Personen, als Institutionen, Regeln etc.

Vertrauen ist ein temporäres Angebot der Komplexitätsöffnung durch einen ,gate keeper': entweder einer Person, der man vertraut, einer Institution oder eines sozialen Mechanismus'. Vertrauen hat - als moderne Form - keinen Stabilitätswert, sondern eine Ankopplungsfunktion (vgl. im Folgenden Priddat 2005: Kap. 3.2).

Vertrauen ist ein ,gate keeper; ${ }^{6}$ aber danach muss man selber Erfahrungen machen. Vertrauen bekommt eine transitorische Funktion: hochbedeutsam für die Eröffnung 
eines neuen Spiels, aber riskant dann, wenn es treu beibehalten wird. Dann wirkt Vertrauen innovationshemmend, d. h. handlungsoptionseinschränkend. Denn die spezifische Bedeutung des Vertrauens, hohe Eingangssicherheit zu bieten, nimmt im Laufe der Zeit ab, wenn es dogmatisch wird, d. h. auf Interpretationsschemata beharrt, die nicht mehr situationsgemäß sind. Vertrauen - um dies zu präzisieren - hat in der Moderne eine Verlaufsgestalt: Anhaltendes Vertrauen kann Erfahrungswerte der Vergangenheit unproduktiv in die Zukunft zerren und Missdeutungen produzieren. Dann wird Vertrauen zu einer irrigen Erwartung und kontra-optional (vgl. Bolton/Ockenfels 2006)

Vertrauen - als Vertrauen/Misstrauen-Ambiguität - ist kein Faktor, keine institutionalisierbare Institution, sondern interaktionssensibel: ein hohe Aufmerksamkeit erheischender wechselseitiger Beobacbtungszustand, der um so ausbeutbarer, damit um so enttäuschbarer wird, je stärker das konventionale Moment des Vertrauens Raum greift. Wenn ich glaube, mich nach hinreichender Erfahrung auf jemanden oder auf eine Institution, wirklich verlassen zu können', nimmt meine Aufmerksamkeit ab, und damit die Beobachtung des Anderen oder der Mechanik der Institution. Ich gerate in einen Zustand des nicht mehr kontrollierten Vertrauens, der die alte abendländische Qualität des Vertrauens ausmacht, der aber in hochagilen Interaktionswelten der Moderne strategisch wie taktisch ausnutzbar wird. Auf Vertrauen kann man nicht mehr vertrauen, lautet die Quintessenz, sondern muss es sich ständig wieder erarbeiten, bestätigen lassen, Signalsysteme haben, die darüber aufklären, wie der mögliche Vertrauensstatus ist etc. Vertrauen wird ein kommunikativer Prozess, keine Eigenschaft, die dem Charakter von Personen zugeschrieben werden kann.

Es ist ein schnell generiertes Vertrauen, das auch schnell wieder verflüchtigt. Vertrauen, können wir zwischendurch resümieren, verliert seine alteuropäische Stabilisationsgarantie. Wir vertrauen nicht mehr erprobten, ausgewählten Vertrauenspersonen oder Vertrauensmechanismen, sondern wir vertrauen sehr viel mehr und sehr viel schneller unbekannten Personen oder Mechanismen, die uns hoch divers und hoch projektspezifisch angeboten werden. Das ist in einer auf Innovation gepolten Gesellschaft und Wirtschaft nichts Ungewöhnliches. Nicht mehr das über lange Zeit ausgebildete Vertrauen ist maßgebend, sondern ein Vertrauen, das in kurzer Zeit gebildet werden kann: über besondere Zeichenbildungen, Markierungen, Auszeichnungen, Kommunikationen.

Das scheint in der Finanzkrise einerseits gelungen zu sein: die sich ausweitende Kommunikation der positiven Zeichen - der Signifikantenketten (vgl. Pretting 2009: Sp. 1). Zum anderen aber haben wir es mit overconfidence zu tun, einer Überbewertung der Vertrauensbeziehungen der Anleger zu ihren Beratern: ein Rückfall in vormoderne trust-relations.

Dieser eher taktische Umgang mit dem Vertrauen herrscht im Finanzmarkt vor. Man delegiert dem Bankberater/Fondmanager etc. seine Entscheidung, um das Risiko nicht kalkulieren zu müssen (weil es zudem komplex und nicht privat einschätzbar ist). Der Vertrauensakt setzt voraus, dass der Bankberater das einschätzen kann, was der Privatmann nicht kann oder will. Nicht einschätzen kann der Investor, dass der Bankberater unter Anreizdruck steht, bestimmte Produkte zu verkaufen, weil sie höhere Gewinnspannen haben (und deshalb höhere Risiken). Und er kann nicht einschätzen, 
dass ein Reputationswettbewerb läuft, der nur so stark expandieren kann, wenn man das Vertrauen der Investoren ausbeutet (bei Lehmann hießen die Zertifikatekäufer im internen Diskurs A und D [Alte und Dumme]). Die Vertrauensbeziehung ist unter diesen Bedingungen von vornherein asymmetrisch. Wenn der Bankberater zudem noch glaubt, dass das System, das er vertritt, , sicher' sei, rationalisiert er lediglich den Druck, unter dem er steht, aber erweist sich faktisch als inkompetent (da er ja Nutzen und Kosten erwägen soll).

Beide vertrauen - der Investor dem Berater, der Berater dem System -, aber ihre Beziehung erfüllt nicht mehr die Kriterien einer Vertrauensbeziehung. So wie sie sich beide über die Risiken täuschen, täuschen sie sich zudem und zusätzlich über das, worauf sie sich einlassen: Sie rutschen in einen Modus alten Vertrauens. Der Berater glaubt wenigstens noch, dass das System als Institution funktioniert; aber der Investor vertraut, neben der Bank als Institution, einer Person. Man verhält sich dem Berater loyal gegenüber, will ihn auch nicht enttäuschen. Damit wandelt sich der letzte Rest an Skepsis in Psychologie.

\section{Vertrauen als moralische Instanz?}

Und in diesem Kontext wird an Vertrauen als moralische Kategorie erinnert: Begriffe wie Ehrbarkeit, Zuversicht, Treue etc. werden memoriert und als Zulassungskriterien für das unbedingte Vertrauen aufgerufen. Neben der neuen Dimension des glücksverheißenden Wachstums der Wirtschaft - die financial markets waren die neuen Wertschöpfungsarenen - kommt eine alte Dimension zum Zuge: Wir vertrauen Personen, die uns bisher immer gut beraten haben - in völliger Verkennung der Systemeigenschaften des Kapitalismus. Die Investoren legten Erwartungen an die Personen, die diese nicht erfüllen konnten (cultural dissonance).

Sind andererseits die Banker, die diese hohen Renditen anbieten, unmoralisch? Sie vertrauen ebenfalls: und zwar dem System. Nur so lässt sich erklären, dass sie ihre jeweilige Kompetenz, Chancen und Risiken angemessen einzuschätzen, beiseite drängten. Sie glaubten an eine kulturelle Änderung des Kapitalismus, der, endlich bei sich angekommen, ein neues Reich der Mehrwertschöpfung kreiert hatte, das goldene Zeiten versprach. Dass dieses Vertrauen in eine segensbringende Zukunft so ausgeprägt aufschäumte, ist das eigentlich interessante Kulturphänomen (nachdem wir es in der new market-Internet-Krise um 2000 ja bereits schon einmal erlebt hatten). Ich nenne dieses Phänomen ,normative Erwartungsdogmatik': Das Wachstum, die Wertschöpfung hat zu geschehen!

Das Vertrauen ist eine Institution, die die Skepsis ebenso ausblendet wie eine rationalere Risikoeinschätzung. Vertrauen funktioniert wie eine Regel, der man unbefragt folgt. Vertrauen ersetzt keine Moral, bekommt aber die Rolle eines Äquivalents: Man vertraut, weil man das im Vertrauen ausgeführte Handeln für legitim und rechtfertigbar hält, ohne es vor sich selber rechtfertigen zu müssen. Es ersetzt die eigene Urteilskraft, hat als Regel eine eigene normative Kraft: ,Es wird schon richtig sein', denn ,alle tun es jac. Wir unterschätzen leicht diese soziologische Dimension. Alle beobachten sich untereinander, wie sie handeln, und kopieren das, was sie beobachten, solange es nicht irritiert wird. Die Regel, die sich durchsetzt, verstärkt das kopierende Verhalten. Man vertraut schlicht dem, was alle machen. 
Die Finanztransaktionen sind somit zweipolig strukturiert: zum einen die großen Optionen, mit ihrem hoffnungsstrategischen Gewährleistungs- und Sicherheitsglauben im Hintergrund. Zum anderen die Quasi-Legitimation durch die Vertrauensnormen. Vertrauen hat grundsätzlich positiven Wert: Immer dort, wo vertraut wird, wird zugleich sozial gerechtfertigtes Handeln vermutet. Vertrauen schaltet Misstrauen aus, macht in gewissem Sinne blind.

Auf die Frage, ob in der Finanzkrise mehr Misstrauen nicht gesünder gewesen wäre, antwortet Guido Möllering, Soziologe am MPI Köln:

„Im Nachhinein: Ja, klar. Doch beim Vertrauen geht es um Ungewissheit und Verwundbarkeit. Man kann daher nur schwer im Voraus den Punkt bestimmen, ab dem zu viel vertraut wird. Zur Finanzkrise hat wohl auch geführt, dass zu viele Beteiligte den neuen Produkten und den damit verknüpften schönen Aussichten allzu gern vertrauen wollten. Vertrauen ist immer auch ein Stück weit Selbsttäuschung“" (Möllering 2009).

Vertrauen leiht sich gleichsam moralische Temperatur, ohne selber Moral zu sein, da es den Nimbus des Gerechtfertigten hat. Indem die Anleger in der dynamischen Finanzwelt ihre Risikoskepsis dämpfen, schalten sie zugleich moralische Bremsen aus. Das Vertrauen erlaubt ihnen, das Risiko in Hoffnung zu übersetzen. Indem die Anbieter das Vertrauen nutzen, um ihre profitablen Transaktionen durchzusetzen, beuten sie - oft ohne es zu wissen - die moralische Struktur der Vertrauensbeziehung aus. Indem sie behaupten, ,vertraue mir, ich habe dich immer gut beraten', suggerieren sie ein rechtfertigbares Handeln, dessen Legitimation nicht mehr befragt werden muss.

So wie das Vertrauen für Transaktionen genutzt wurde, denen man eigentlich nicht vertrauen kann, so wirkt das neue Prinzip Hoffnung als remake des alten kapitalistischen Versprechens, dass jeder seine Wohlfahrt erhöhen könne: in den USA vor allem Einkommensschwache, denen Hausbesitz schaffende Kredite (subprimes) mit dem Argument angedient wurden, dass sich nun endlich auch die Ärmeren am Reichtum beteiligen könnten. Das Argument ist wohlfahrtsmoralisch temperiert, allerdings unter Weglassung der Risiken, die eine höhere Verarmung bedeuten können.

Es zeigt sich eine - mit Glückserwartungen gekoppelte - moralische oder quasimoralische Legitimation, welche die ökonomische Kompetenz der Beurteilung dieser Transaktionen verdünnte. Es zeigt sich aber auch, dass Risikoeinschätzungen zwar Teile der Ausrüstung der rational choice oder ökonomischen Vernunft darstellen, aber letztlich kulturell oder moralisch/quasi-moralisch konfundiert sind. Nicht der ökonomische Kalkül, sondern seine kulturelle Interpretation in einer Vertrauenskultur und in einer historischen Glückserwartung entscheiden über die Systemeffekte. Es kommt darauf an, wie man in einer Kultur Risiko interpretiert und kommuniziert (vgl. Beckert 2009).

Die moralische Kritik - Gier, Hybris, Verantwortungslosigkeit etc. - trifft nicht den Handlungskern in ökonomischen Systemen. Moral setzt voraus, dass es anerkannte Regeln gibt, die zu ignorieren allen anzeigt, dass hier erwartete Verhaltensmuster ungültig sind, weil sie Andere schädigen oder unbillig übergehen. Man verhält sich ,unanständig', d. h. nicht standesgemäß. In einer modernen ständelosen Gesellschaft hieße das übersetzt: nicht regelkonform. Doch welche Regeln gelten? 
Wenn die Regeln als Handlungsgewohnheiten oder informelle Institutionen kulturell ausgeprägt sind, können wir nicht erwarten, dass das individuelle Gewissen moralisch schlägt, oder dass ältere Tugendkataloge aufgeblättert werden, um Einhalt zu gebieten. Solche moralischen Regeln haben keine systematische Geltung, auch wenn sie individuell vorkommen. Deshalb sind Vorschläge wie ,Demut', ,Anstand' oder generell ,Moral' ${ }^{`}$ zwar Erinnerungsposten an ideale Verhaltensmodalitäten, treffen aber nicht den Kern gelebter Geschäftskulturen. Moral wird kommuniziert, wenn diese Kulturen abbrechen; Moral hat dann Indikatorfunktion, sie weist auf Verhaltensunklarheiten hin, ohne selber Klarheit zu schaffen (vgl. Priddat 2007).

Praktisch reagieren die von der Finanzmisere getroffenen Staaten mit neuen Regeln, nicht mit der Aufforderung, tugendhaft zu werden. Regeln sind informelle Institutionen, welche die wechselseitigen Handlungserwartungen konventionalisieren. Das lässt sich nicht in Moral übersetzen, aber in ihr funktionales Äquivalent. Regeln sollen neues Vertrauen herstellen. Das klingt positiv. Vertrauen ist aber potentiell wieder ausbeutbar, und zwar systematisch deshalb, weil es die skeptische Rationalität mindert. Es tut mir Leid für die Liebhaber des Ethos des ,guten Kaufmannes', wenn ich an dieser Stelle statt Moral nur Skepsis empfehlen kann.

Oder, um es pointierter zu sagen: Wenn sich alle rational im Sinne ökonomischer Rationalität verhalten hätten, hätte die damit einhergehende Risikokompetenz das Ausmaß dieser Krise nicht entstehen lassen dürfen. Aber das ist natürlich auch eine idealistische Aussage, denn Märkte funktionieren nicht rational gesteuert (vgl. Shiller 2003). Untersuchen wir fortan besser deren Kulturen, um die Verhaltenspotentiale zu ermessen. Es geht hier weniger um Moral, sondern um ironische Distanz zu VerheiBungen. Analysieren wir nüchtern alle Formen ökonomischer Eschatologie.

Denn in welche Paradoxie begeben wir uns jetzt? Wenn das Vertrauen wieder aufgebaut werden soll, heißt das, dass wir uns wieder in den Zustand der Unschuld begeben, wie vor der Krise?

„Vertrauen als Mittel zum Erhalt der Marktwirtschaft: Vertrauen soll wiederhergestellt werden, indem man denen, die die Missstände geschaffen und damit Misstrauen herbeigeführt haben, Geld gibt?" (o. V. 2008).

Ist diese Rekonstitution von Vertrauen gerechtfertigt? Andere Formen der Rekonstitution sind eher regelorientiert, d. h. sie bevorzugen die Kontrolle des Bankgeschäfts (vgl. Siebert 2008; Weder di Mauro 2009):

„Ich bin sehr skeptisch gegenüber Kontrollreflexen, die das verlorene Vertrauen ersetzen wollen und damit seine Rückkehr doch eher erschweren. Soll Kontrolle Vertrauen zurückbringen, müssen erstens die Kontrollierten die Kontrollen akzeptieren und zweitens Freiräume bleiben, in denen sie zeigen können, dass sie freiwillig verantwortungsvoll und solidarisch sind. Andererseits müssen wir uns im Finanzsektor vielleicht doch für mehr Kontrolle entscheiden, weil ein hohes $\mathrm{Maß}$ an Vertrauen dort zu voraussetzungsvoll und gefährlich ist" (Möllering 2009).

Dass Vertrauen riskant sein kann, ist eine unterbewertete Kenntnis, konträr zur moralischen Aufwertung, die das Vertrauen gerade erfährt. Möllering nennt das hohe Maß an Vertrauen im Finanzsektor „,voraussetzungsvoll und gefährlich“: 
- Voraussetzungsvoll heißt: Nicht jeder, der die komplexe Materie nicht versteht, kann darauf vertrauen, dass es anderen anders geht. Auch nicht denen, denen er vertraut im Anlagegeschäft.

- Gefährlich heißt: Hier sind Risiken im Spiel, die nicht nur individuelle Vermögensenttäuschungen bedeuten können, sondern systemische Risiken (Risiken zweiter Ordnung bzw. die Differenz zwischen betriebswirtschaftlichen und volkswirtschaftlichen Risiken [vgl. Hellwig 2008]).

Beide Risiken lassen keinen Weg zurück in ein wiederzugewinnendes Vertrauen einer Klient/Berater-Beziehung. Es geht nur mehr über ein third party enforcement: über eine dreigliedrige Relation Klient/Berater/Aufsicht (sanktionsbewehrt). Wieso sollte man einer zweigliedrigen Klient/Berater-Relation, d. h. einer Form des Vertrauens trauen, das sich als überfordert gezeigt hat?

Homann hat Recht: Unter dem Wettbewerbsdruck wäre es für keinen Banker einfach gewesen, das Vertrauen, das die Anleger in sie gesetzt hatten, dadurch zu rechtfertigen, indem sie ihrer Klientel abgeraten hätten, die Papiere zu kaufen, die heute als ,toxisch' bezeichnet werden. In diesem Sinne hatten sie gar keine Chance, mit dem ihnen entgegengebrachten Vertrauen verantwortungsvoll umzugehen (nicht einmal dann, wenn sie ausgestiegen wären, denn dann wären sie ja auch aus ihrer Beziehung mit den Klienten ausgestiegen). Wir haben es mit einer scharfen Asymmetrie zu tun: Die Anleger vertrauen, die Berater können es, wegen ihrer Wettbewerbszwänge, nicht erwidern (nur rhetorisch, aber darauf kommt es in Verhandlungen ja erst einmal an). Was gewährleistet uns, dass es ein nächstes Mal ,besser' geht? Was heißt hier besser? Wenn wir wieder vertrauen? Aber die Asymmetrie ist ja nicht aufgehoben. Wann können wir darauf vertrauen, dass ein Bankberater uns verantwortungsvoll abrät, eine ganze Klasse von Papieren zu kaufen, weil die Risiken zu hoch seien (obwohl für den Verkauf gerade dieser Papiere seine persönliche Prämie auch sehr hoch sein würde)?

\section{Literaturverzeichnis}

Beckert, J. (2009): Koordination und Verteilung. Zwei Ansätze der Wirtschaftssoziologie, MPIfG Discussion Paper 09/2.

Bolton, G. E./ Ockenfels, A. (2006): The Limits of Trust in Economic Transactions. Investigations of Perfect Reputation Systems, Working Paper Universität Köln, 13. Okt. 2006.

Coval, J. D./ Jurek, J. W./ Erik, S. (2009): The Economics of Structured Finance, in: Journal of Economic Perspectives, 23(1), 3-25.

Hellwig, M. (2008): Wenn die Interessen verwischen, in: FAZ, Nr. 274, 22.11.2008, 13.

Homann, K. (2009): Wettbewerb ist janusköpfig, in: Spiegel Online, 07.03.2009.

Möllering, G. (2009): Vertrauen ist immer auch Selbsttäuschung, in: Spiegel Online, 15.02.2009.

o. V. (2008): Finanzkrise Vertrauen, in: Socanalytica (Blog), 26.10.2008, 11:31.

Pretting, G. (2009): Traurige Märkte, in: Die Zeit, Nr. 18, 23.04.2009, 45.

Priddat, B. P. (2005): Unvollständige Akteure - Komplexer werdende Ökonomie, Wiesbaden: VS-Verlag.

Priddat, B. P. (2007): Moral als Indikator und Kontext von Ökonomie, Marburg: Metropolis. 
Shiller, R. J. (2003): From Efficient Markets Theory to Behaviorial Finance, in: The Journal of Economic Perspectives, 17(1), 83-104.

Siebert, H. (2008): Ein Regelwerk für die Finanzmärkte, FAZ, Nr. 250, 25.10.2008, 11.

Strack, F. (2008): Psychologie der Finanzkrise. Die verrückte Mär vom irren Markt, in: Spiegel Online, 23.10.2008.

Weder di Mauro, B. (2009): Ein Gesamtkonzept für die Rettung der Banken fehlt, FAZ, Nr. 33, 09.02.2009, 15 . 